\title{
Preparation of Peripheral Blood Mononuclear Cell Pellets and Plasma from a Single Blood Draw at Clinical Trial Sites for Biomarker Analysis
}

\author{
Paola Marco-Casanova ${ }^{1}$, Natalia Lukashchuk ${ }^{1}$, Benedetta Lombardi ${ }^{1}$, Veerendra Munugalavadla $^{2}$, Melanie M. \\ Frigault $^{3}$, Elizabeth A. Harrington ${ }^{1}$, J. Carl Barrett ${ }^{3}$, Andrew J. Pierce ${ }^{1}$ \\ ${ }^{1}$ Translational Medicine, R\&D Oncology, AstraZeneca ${ }^{2}$ Acerta Pharma, AstraZeneca Group ${ }^{3}$ Translational Medicine, R\&D Oncology, AstraZeneca
}

\section{Corresponding Authors}

Paola Marco-Casanova

paola.marco-casanova@astrazeneca.com

Andrew J. Pierce

apierce@crescendobiologics.com

\section{Citation}

Marco-Casanova, P., Lukashchuk, N., Lombardi, B., Munugalavadla, $\mathrm{V}$.,

Frigault, M.M., Harrington, E.A.,

Barrett, J.C., Pierce, A.J. Preparation

of Peripheral Blood Mononuclear Cell Pellets and Plasma from a Single Blood Draw at Clinical Trial Sites for Biomarker Analysis. J. Vis. Exp. (169), e60776, doi:10.3791/60776 (2021).

\section{Date Published}

March 20, 2021

DOI

$10.3791 / 60776$

URL

jove.com/video/60776

\section{Abstract}

Analysis of biomarkers in peripheral blood is becoming increasingly important in clinical trials to establish proof of mechanism to evaluate effects of treatment, and help guide dose and schedule setting of therapeutics. From a single blood draw, peripheral blood mononuclear cells can be isolated and processed to analyze and quantify protein markers, and plasma samples can be used for the analysis of circulating tumor DNA, cytokines, and plasma metabolomics. Longitudinal samples from a treatment provide information on the evolution of a given protein marker, the mutational status and immunological landscape of the patient. This can only be achieved if the processing of the peripheral blood is carried out effectively in clinical sites and samples are properly preserved from the bedside to bench. Here, we present an optimized general-purpose protocol that can be implemented at clinical sites for obtaining PBMC pellets and plasma samples in multi-center clinical trials, that will enable clinical professionals in hospital laboratories to successfully provide high quality samples, regardless of their level of technical expertise. Alternative protocol variations are also presented that are optimized for more specific downstream analytical methods. We apply this protocol for studying protein biomarkers against DNA damage response (DDR) on X-ray irradiated blood to demonstrate the suitability of the approach in oncology settings where DDR drugs and/or radiotherapy have been practiced as well as in preclinical stages where mechanistic hypothesis testing is required.

\section{Introduction}

Drug development aims to deliver new therapeutics addressing unmet medical needs and more targeted, personalized medicine. Multiple drug mechanisms are under active investigation including enzymatic inhibition such as kinase $^{1}$, protease ${ }^{2}$, or poly (ADP-ribose) polymerase (PARP) inhibitors ${ }^{3}$, protein degraders ${ }^{4}$, therapeutic antibodies ${ }^{5}$, and 
antibody-conjugated drugs (ADCs $)^{6}$, among many others. An example of the efforts to obtain better treatments in oncology is the use of kinase inhibitors with the goal of stopping the signaling cascades that keep cancerous cells proliferating ${ }^{1,7}$. Measuring the levels of substrate phosphorylation specific to those kinases is the best pharmacodynamic biomarker to quantify the mechanism of action of these inhibitors ${ }^{8}$. Other drugs may modulate the expression of a given protein, and in that case being able to quantify the changes in concentration of their target protein longitudinally throughout the course of treatment is paramount. Therefore, independent of the characteristics of a drug or a pathology, evaluation of biomarkers to establish the pharmacokinetics (PK)/ pharmacodynamics (PD) relationship between drug exposure and target modulation is the best practice in early clinical development and enables the determination of a safe and tolerated pharmacologically active dose/schedule ${ }^{9}$.

While in oncology clinical development, biomarker analysis in biopsies might be the best setting to establish proof of mechanism of a drug, the number of available biopsies in a trial is usually quite limited ${ }^{10,11}$. Alternatively, peripheral blood samples are highly valuable to clinical trials because they involve a minimally invasive procedure, are quick and easy to obtain facilitating longitudinal analysis, are less expensive than biopsies and provide vast information for real-time monitoring of the outcome of a treatment. An additional benefit to assessing PD biomarkers in peripheral blood is the capacity to use the biosample to also quantify PK allowing exactness in determining PK/PD quantitative relationships and subsequent PK/PD modeling ${ }^{12,13}$. Peripheral blood mononuclear cells (PBMCs) from whole blood can be isolated to study protein markers, which experience either changes in their expression level or in their post-translational modifications. In addition,
PBMCs can be used for immunophenotyping purposes ${ }^{14,15}$, immune functionality assays such as assessing antibodydependent cellular cytotoxicity $(A D C C)^{16}$ and epigenetic analysis through RNA isolation. Likewise, plasma from whole blood can be used to quantify cytokines to characterize the immunological response of a patient, to perform metabolic studies, and also to isolate and sequence circulating tumor DNA (ctDNA) for monitoring the clonal evolution of disease under selection from the therapeutic agent, frequently providing a mechanistic basis for treatment resistance ${ }^{17,18,19}$ enabling development of subsequent generations of therapeutics ${ }^{20}$. Finally, isolation of circulating tumor cells (CTCs) from peripheral blood allows for the evaluation of disease progression by longitudinal enumeration, DNA/RNA sequencing and protein-biomarker analysis ${ }^{21}$. Although this isolation is compatible with the protocol described herein ${ }^{22}$, the low abundance of CTCs in many cancer types and early stages of the disease makes the use of specialized tubes more suitable for minimizing CTC degradation ${ }^{23}$.

In recent years, the use of liquid biopsies has improved the information obtained in clinical trials and PBMC collections have been included in many studies to monitor target engagement and proof of mechanism either directly in tumor cells for some types of hematological malignancies, or on the PBMCs themselves as PD surrogates of tumor cells $24,25,26$. The preparation of high-quality samples positively impacts determining the safest and most efficacious treatment for a given pathology but in our experience, the quality of PBMC preparations obtained from different clinical sites has been subjected to wide variability in quality resulting in samples that are not fit for the purpose of downstream analysis. This has 
impacted the amount of PD data that could be collected from those studies.

Here we describe in detail an easy to follow protocol that shows how to efficiently isolate both PBMCs and plasma samples from a single blood draw in a clinical setting. The protocol is based on the instructions provided by the manufacturer of the mononuclear cell preparation tubes, which incorporates modifications where real-world experience has highlighted difficulties in protocol execution as reported by clinical sites, such as centrifugation issues, processing delays and sample transfer to cryovials. There are alternative commercially available methods to the use of mononuclear cell preparation tubes based on density gradient separation using polysaccharide solutions with or without a barrier that separates the solution from the blood ${ }^{27}$. If the relevant clinical site is already well-experienced in these alternative methodologies, this protocol can be acceptably substituted with these. In such cases, two factors can be considered: some alternative methods require a transfer of whole blood from a collection tube to a separate preparation tube where an additional transfer of human primary biological material may present a slightly increased safety risk, and the success of methods without barriers separating the blood from the polysaccharide solution relies on critical steps such as layering the blood sample on the density gradient medium requiring development of a refined level of technical expertise not always found in a hospital laboratory setting. The above points notwithstanding, the overall viability and cell recovery are comparable between these techniques ${ }^{15,28}$. Choice of the methodology is, therefore, somewhat dependent upon prior technical experience, but in our hands, mononuclear cell preparation tubes can be used successfully in a broad clinical context and are our, otherwise, recommendation.
While one endpoint of this protocol is to produce PBMC pellets for further processing into lysates, other final applications of the PBMC collection could be implemented, such as isolation of nucleic acids, or producing PBMC smears or PBMC blocks suitable for immunohistochemistry (IHC) methods. Importantly, since each biosample taken from patients represents an invasive procedure on at least some level, this protocol maximizes the useful material from each sample by also isolating plasma which can be used for cytokine analyses, metabolomic studies or ctDNA sequencing.

The analysis of peripheral biomarkers in oncology trials is one of the many applications of PBMC lysates. One example is the evaluation of the DNA damage response (DDR) in treatments such as chemotherapy, radiotherapy or the use of inhibitors of enzymes involved in the DDR such as the phosphatidylinositol-3 kinase-related kinases (PIKKs) ${ }^{7}$ and PARPs $^{3,13}$. The aim of these treatments is to increase DNA damage in proliferating cells, which generates high toxicity in cells with impaired DDR mechanisms and cell cycle checkpoints, such as cancer cells. Here we present an example with a study of DDR biomarkers in peripheral blood subjected to X-rays.

\section{Protocol}

Informed patient consent and full compliance with relevant national ethics requirements in each jurisdiction e.g., the Human Tissues Act (HTA - United Kingdom, 2004) and the Health Insurance Portability and Accountability Act (HIPAA - United States, 1996) are mandatory. Be certain to have fully documented ethics approval before beginning any work on human-derived materials. Blood used in the optimization of this protocol was provided with appropriate consent from the Volunteers Advancing Medicine Panel (VAMP) (ethics reference 16/EE/0459, study CRF494, sub study 
001) run by the NIHR Cambridge Clinical Research Facility, Cambridge, United Kingdom, under a Human Biological Samples supply agreement with the National Institute for Health Research (NIHR) Cambridge Biomedical Research Centre. The AstraZeneca Biobank in the UK is licensed by the Human Tissue Authority (License No. 12109) and has National Research Ethics Service Committee (NREC) Approval as a Research Tissue Bank (RTB) (REC No 17/ NW/0207).

\section{General preparation guidance}

NOTE: All work with unfixed human material such as the blood, plasma and PBMCs in this protocol must operate under the assumption that these materials may carry potentially infectious agents and so must be performed under suitable biosafety precautions. For patients that have been tested as negative for known pathogens, do not assume samples are non-infectious and so suitable safety precautions must still be applied. Waste products generated from these materials should be treated with the same biosafety precautions and disposed according to local rules.

1. Choose between the two types of mononuclear cell preparation tubes that utilize either sodium citrate or sodium heparin as anticoagulants. For many downstream applications these two anticoagulants can be used interchangeably but both anti-coagulants should be tested before selecting one for the trial.

2. Label one $8 \mathrm{~mL}$ mononuclear cell preparation tube to be used for the blood collection with the patient's coded ID. Keep the tubes at room temperature $\left(18-25^{\circ} \mathrm{C}\right)$.

3. Store $1 \times \mathrm{PBS}$ at room temperature $\left(18-25^{\circ} \mathrm{C}\right)$. Use 30 $\mathrm{mL}$ per preparation.
4. Make sure tubes for separate plasma samples and the cryovial for the PBMC sample are accurately labeled with unique identifiers, as specified in the appropriate section of the lab manual.

5. If PBMCs are going to be used to monitor phosphorylated proteins, prepare PBS supplemented with phosphatase inhibitors: mix $5 \mathrm{~mL}$ of PBS with $50 \mu \mathrm{L}$ of phosphatase inhibitor cocktail $2+50 \mu \mathrm{L}$ of phosphatase inhibitor cocktail 3. Prepare fresh and keep on ice until use.

6. If PBMCs are going to be cryopreserved, prepare $1 \mathrm{~mL}$ freezing mixture per sample by mixing $90 \%$ FBS $+10 \%$ DMSO.

\section{PBMC collection (Figure 1A)}

1. Draw $8 \mathrm{~mL}$ blood into the mononuclear cell preparation tube using the standard technique described by the manufacturer. Invert the tube gently 8 to 10 times to mix the anticoagulant additive with blood. Do not shake to avoid hemolysis. Record the time at which the blood was drawn.

2. After collection, store the tube upright at room temperature until centrifugation. Process the samples as soon as possible, ideally within one hour of this blood collection but not later than $4 \mathrm{~h}$ post collection. Record the timing when starting the processing of the blood.

3. Immediately prior to the centrifugation remix the blood sample by gently inverting the tube 8 to 10 more times.

4. Centrifuge the tube/blood sample tubes in a horizontal rotor (swing-out head) at 1,500 - 1,800 $\times \mathrm{g}$ for $30 \mathrm{~min}$ at room temperature $\left(18-25^{\circ} \mathrm{C}\right)$. Ensure that all the tubes are balanced properly. 
A)
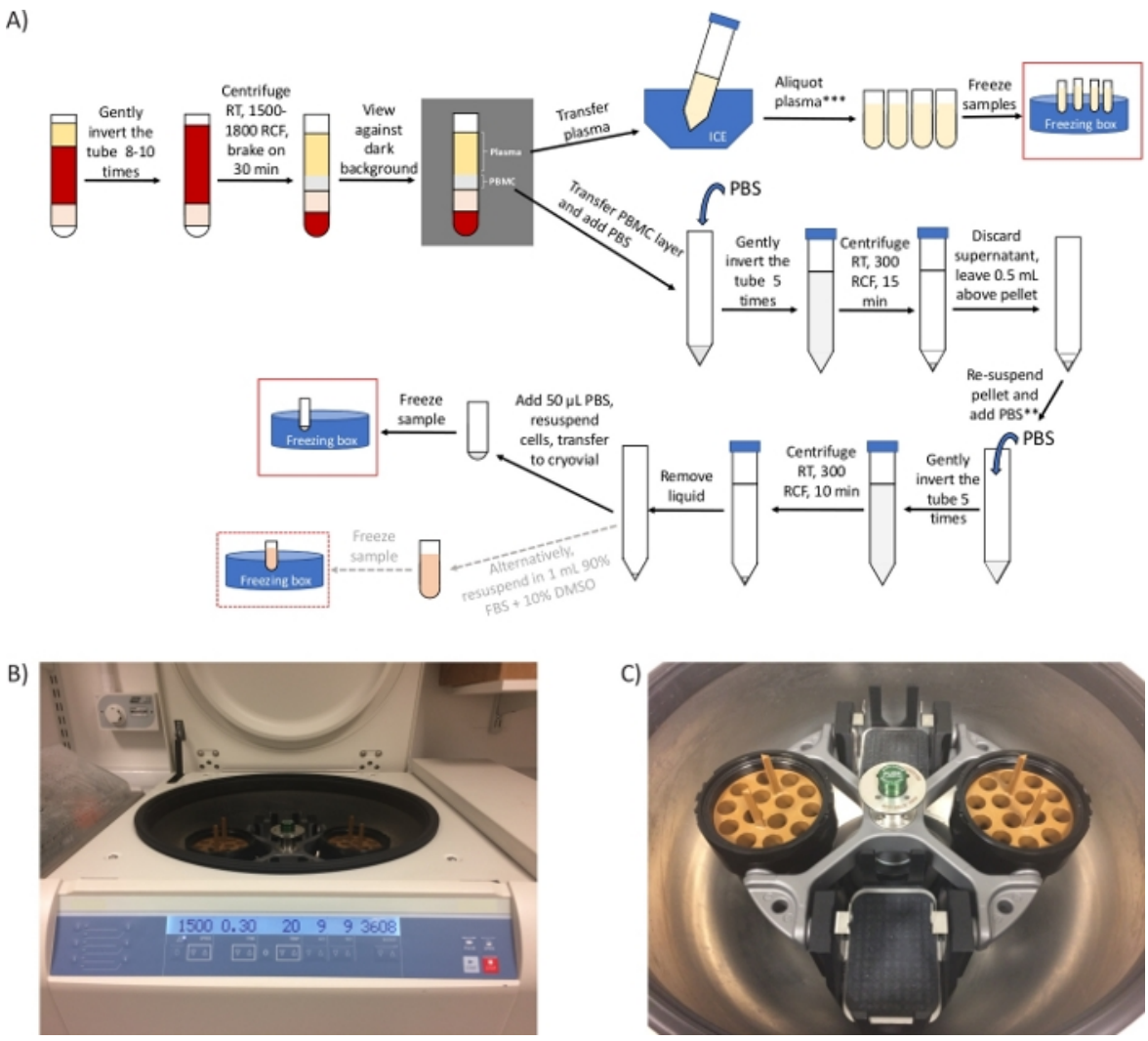

Figure 1: General overview of the protocol and representative images of the centrifuge. (A) Schematic overview of the protocol for the preparation of PBMCs and plasma. *If there are time constraints, step 2.4 can be shortened to 20 min and steps 3.3 to 3.6 can be removed. ${ }^{* *}$ Take an aliquot to count cells, if required. ${ }^{* *} 2$ extra centrifugation steps required for ctDNA analysis/metabolomics (B) image of a swing-out head rotor centrifuge set for step 2.4. (C) Image of the rotor, which includes two buckets containing the adaptors to spin mononuclear cell preparation tubes. Please click here to view a larger version of this figure.

NOTE: $x g$ (also referred to as RCF, relative centrifugal units) and RPM (revolutions per minute) are different units. Be sure to set the centrifuge for $x g$ (RCF). Use the online calculator (see Table of Materials) for the conversion. If only fixedangle rotor is available, perform this step for $10 \mathrm{~min}$ at 1,500 $-1,800 \times g$.
5. After centrifugation, check for the presence of a dark red layer under the barrier (containing mostly red blood cells), and 2 layers above the barrier. The top layer is the plasma (straw-colored) and the whitish layer underneath is the buffy coat containing the PBMCs. These layers can be easily distinguished when viewing the tube against a dark/black background. 

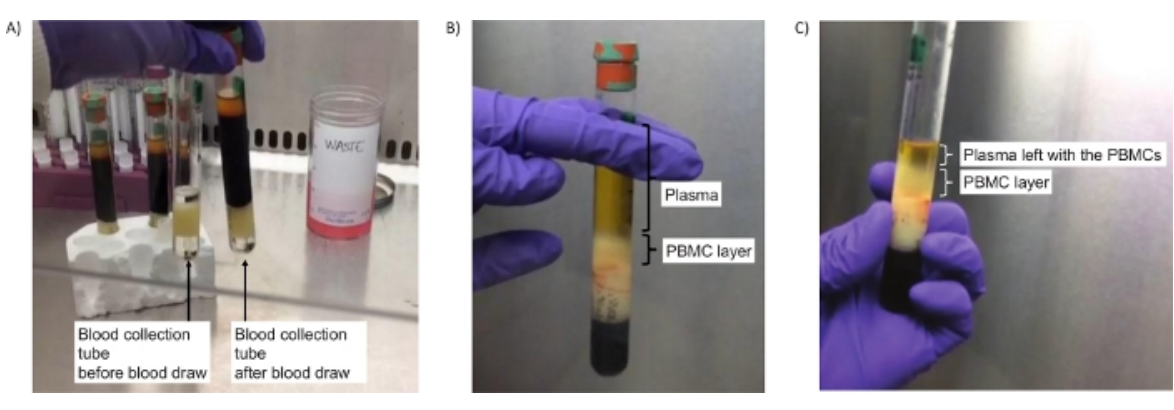

Figure 2: Tubes to isolate PBMCs. (A) Image of the tubes before centrifugation (step 2.4), either empty (left) or containing blood (right). (B) Successful separation of the PBMC layer after centrifugation (step 2.5). (C) Image of the PBMC layer after centrifugation and a bit of plasma left to ensure all PBMCs are collected (step 2.7). Please click here to view a larger version of this figure.

NOTE: If these layers are not visible, this probably indicates an error in setting up the centrifugation units. Ensure the right centrifuge adapter is used and the correct " $\mathrm{x} g$ " are set. Repeat step 2.4.

6. Immediately following centrifugation, use a serological pipette to transfer approximately half of the plasma into a labeled $15 \mathrm{~mL}$ size conical centrifuge tube with cap, while being careful to not disturb the PBMC layer (approximately 4-5 mL). Temporarily store this tube with plasma on wet ice to be used later in the step 4. Set aside for now.

7. Collect the entire PBMC layer with a Pasteur pipette by placing the pipette within the layer of cells and transfer to a different $15 \mathrm{~mL}$ size conical centrifuge tube with cap. It is acceptable to also take a small amount of plasma, if necessary, to completely get all the PBMC layer. The volume is usually $1-2 \mathrm{~mL}$. Immediately continue with the step 3 below.

\section{PBMC washing steps}

NOTE: The purpose of the wash steps is to dilute out and remove residual platelets and plasma from the PBMC pellet. All centrifugation steps should be performed at room temperature $\left(18-25^{\circ} \mathrm{C}\right)$.

1. Add room temperature PBS to the PBMC tube to bring the volume to $15 \mathrm{~mL}$. Cap the tube. Mix cells by gently inverting the tube 5 times.

2. Centrifuge for $15 \mathrm{~min}$ at $300 \times \mathrm{g}$. Note that this is a much gentler centrifugation than the initial centrifugation.

3. Visualize the pellet by viewing the tube against a dark/ black background. Remove the supernatant by vacuum aspiration or with a pipette (Figure 3A). Discard the supernatant leaving a volume of approximately $500 \mu \mathrm{L}$ above the whitish colored PBMC pellet. 

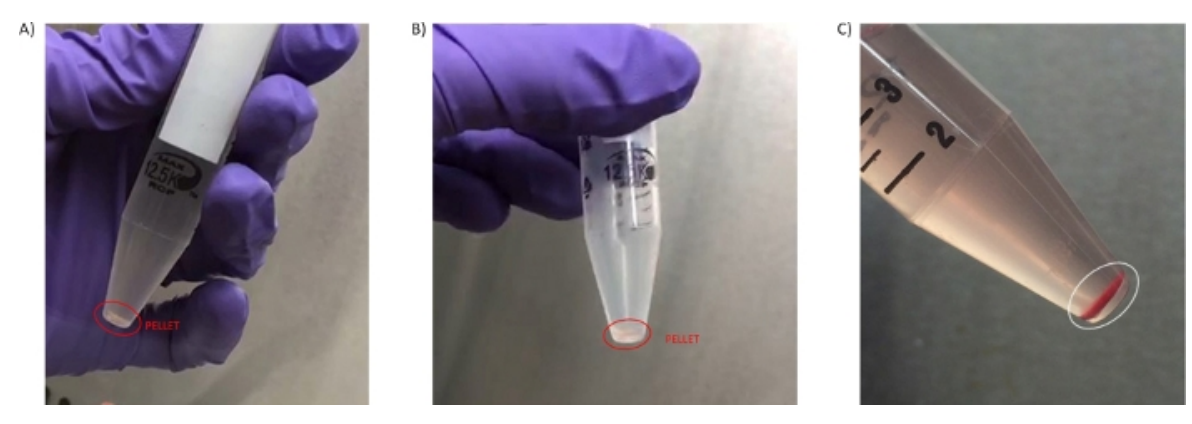

Figure 3: PBMC pellets. (A) Pellet obtained in the first wash step 3.3. (B) Pellet isolated in the second wash (step 3.7). (C) Pellet with high level of hemolysis obtained from blood processed later than $4 \mathrm{~h}$ from the blood draw isolated in the second wash (step 3.6). Please click here to view a larger version of this figure.

4. Resuspend the cell pellet by gently pipetting up and down in the residual supernatant.

5. Add additional $1 \times$ PBS to bring the volume to $10 \mathrm{~mL}$. Cap the tube. Mix the cells by inverting the tube gently $5 x$. If cell counting is required in the study protocol go to step 3.5.1, if not required move directly to step 3.6.

1. Take a $40 \mu \mathrm{L}$ aliquot of the re-suspended cells and mix with $40 \mu \mathrm{L}$ of trypan blue. Count the viable cells using a hemocytometer or an automatic cell counter.

6. Centrifuge the suspension from step 3.5 for $10 \mathrm{~min}$ at $300 \times \mathrm{g}$. Remove as much supernatant as is reasonably possible without disturbing the cell pellet.

7. Carefully remove and discard any remaining supernatant above the cell pellet by pipetting using a fine tip Pasteur pipette or a micropipette without disturbing the cell pellet. Leave little to no liquid above the pellet after completing this step. If the endpoint of this protocol is a PBMC pellet move to step 3.8; if the endpoint is cryopreserved PBMCs go to step 3.10 .
8. Add $50 \mu \mathrm{L}$ of new PBS (or the supplemented PBS prepared in step 1.5 if applicable to your endpoint) to the pellet and pipette up and down gently using a micropipette to homogenously resuspend all the cells. Transfer the entire cell suspension to a labeled $1.5 \mathrm{~mL}$ cryovial and immediately place on wet ice until freezing the samples.

9. Freeze the labeled samples by placing them in liquid nitrogen or directly in dry ice. Cells can also be frozen at $-80^{\circ} \mathrm{C}$ freezer using cell freezing boxes. In this case, prechill the boxes completely in $-80{ }^{\circ} \mathrm{C}$ before adding the cell tubes. Record the time at which cell pellets were frozen. Once cells freeze, store at $-80^{\circ} \mathrm{C}$, ship frozen on dry ice.

10. Optionally, to cryopreserve the PBMCs re-suspend the pellet in $1 \mathrm{~mL}$ of the freezing mixture (step 1.6) and transfer to a labeled cryovial. Continue as described in step 3.9 , but, in this case, it is only acceptable to use cell freezing boxes to freeze down the samples. Transfer to liquid nitrogen for shipping and storage after being in the freezing box for a minimum of $24 \mathrm{~h}$. 


\section{Plasma preparation steps (Figure 1A)}

1. Following $-80{ }^{\circ} \mathrm{C}$ storage of the PBMC pellets, now prepare the plasma aliquot that was temporarily stored on wet ice in step 2.6. Perform the following centrifugation steps to clarify the plasma if the purpose of the sample is ctDNA analysis, otherwise move directly to step 4.2.

1. Centrifuge the plasma for 10 min at $1,600-2,000 x$ $g$ at $4-8{ }^{\circ} \mathrm{C}$ in a fixed-angle rotor (or $15 \mathrm{~min}$ in a swing-out rotor).

2. Carefully pipette off the plasma supernatant, taking care not to disturb any pellet and transfer to a new $15 \mathrm{~mL}$ tube. Discard the tube containing the pelleted material.

3. Centrifuge the plasma for $10 \mathrm{~min}$ at $1,600-2,000 x$ $g$ at $4-8{ }^{\circ} \mathrm{C}$ in a fixed-angle rotor (or $15 \mathrm{~min}$ in a swing-out rotor).

4. Carefully transfer the plasma supernatant to a new $15 \mathrm{~mL}$ tube, being sure not to disturb any pelleted material. Discard the tube containing the pelleted material.

NOTE: If a refrigerated centrifuge is not available, keep cells on ice for 5 min between each spin, or centrifuge samples in a cold room.

2. Transfer plasma in $1 \mathrm{~mL}$ aliquots into 5 fresh $2 \mathrm{~mL}$ microtubes. Use fewer than 5 vials if there is not enough plasma to fill 5 vials with $1 \mathrm{~mL}$ aliquots and it is expected that the last vial will contain less than $1 \mathrm{~mL}$ plasma. If there is more than $5 \mathrm{~mL}$ plasma, discard the remainder. Record the total volume of plasma in each tube.

3. Immediately freeze the plasma aliquots upright, by storing them at $-80{ }^{\circ} \mathrm{C}$.

\section{Sample shipment}

1. Ship both PBMC pellets and plasma samples on dry ice.

2. Ensure that the sample is not thawed before and during shipment. Pack sufficient dry ice with the samples to ensure they remain frozen for the entirety of the shipping process, considering possible delays that may occur in transit.

\section{Whole blood irradiation and western blot analysis of DDR biomarkers (Figure 4A)}

NOTE: This is an ex vivo treatment that will not be required in most cases in the clinic, but these experiments are a valuable exploratory strategy to find suitable clinical biomarkers. Blood samples should be treated as soon as possible upon collection to ensure best results.

1. Warm up the X-ray cabinet. Label three $15 \mathrm{~mL}$ tubes as $0,0.2$ and $7 \mathrm{~Gy}$, respectively.

2. Take three mononuclear cell preparation tubes containing freshly drawn blood from a single individual and after gently inverting the tubes 8 to 10 times transfer the bloods to the three $15 \mathrm{~mL}$ tubes.

3. Place the $0.2 \mathrm{~Gy}$ tube in the X-ray cabinet, close the door and apply 0.2 Gy dose to the tube by selecting the shelf number and dose. Apply $7 \mathrm{~Gy}$ radiation dose to the $7 \mathrm{~Gy}$ tube by adjusting the dose selected in the cabinet.

4. Incubate the three tubes at $37^{\circ} \mathrm{C}$ for one hour.

5. Transfer the blood back to the mononuclear cell preparation tubes and carry out the PBMC preparation protocol as for a clinical setting from step 2.4 to step 3.8 .

6. Add a volume of lysis buffer supplemented with protease and phosphatase inhibitors equal to the cell pellet volume 
(in this case $70 \mu \mathrm{L}$ of RIPA buffer) to each of the cell pellets, pipette up and down and incubate on ice for 10 min. Sonicate the samples if a sonicator is available (3 cycles, 30 s ON / 30 s OFF, $4{ }^{\circ} \mathrm{C}$ ), alternatively syringe the samples to break the nucleic acids to eliminate viscosity in the sample.

7. Centrifuge the samples for $10 \mathrm{~min}$ at $\geq 15,000 \times \mathrm{g}$, at $4{ }^{\circ} \mathrm{C}$. Transfer each supernatant to a new $1.5 \mathrm{~mL}$ tube, qualitatively assess the level of hemolysis (visual inspection) and measure the protein concentration by any preferred method.

8. Mix $40 \mu \mathrm{g}$ of total lysate with sample loading buffer containing SDS and sample reducing agent. Boil samples for $5 \mathrm{~min}$ in a heat block.

9. Load $20 \mu \mathrm{g}$ of each sample per lane in duplicate in a $4-12 \%$ bis-tris protein gel and run an SDS-PAGE.

10. After separation, transfer the proteins to a nitrocellulose membrane using a commercially available system $(20 \mathrm{~V}$, $10 \mathrm{~min}$ ) and block with $5 \%$ milk in TBST.

11. Cut the membrane at the relevant molecular sizes and incubate with the primary antibodies overnight at $4{ }^{\circ} \mathrm{C}$ (see Table of Materials for antibodies and dilutions).

12. Remove the primary antibodies and wash the membranes 3 times with TBST for 5 minutes at room temperature. Incubate with the HRP-conjugated secondary antibodies for 45 minutes at room temperature.

13. Wash 3 times with TBST for 5 min.

14. Apply the ECL reagent and analyze the images obtained relative to HRP signal.

\section{Representative Results}

To improve the quality of PBMC preparations in our clinical trials, we have generated a protocol with concise, clear steps that can be followed by hospital laboratory professionals, independent of their molecular biology background and laboratory skills. We have adapted the manufacturer's protocol incorporating modifications on those steps where execution issues have been identified or reported from clinical sites involved in various multi-center clinical trials. However, the protocol can be further optimized to meet specific requirements, such as time constraints in the clinical site or type of downstream analyses (see Supplementary File). We demonstrate that the DDR can be analyzed in PBMCs by looking at specific biomarkers upon DNA damage generated by radiation.

The most common queries we have received from clinical sites relate to the centrifugation steps, which directly impact being able to successfully isolate PBMCs and obtain the final PBMC pellet. Using the appropriate type of swing-out head rotor centrifuge (Figure 1B,C) is the key to the success of the protocol. However, when only a fixed-angle rotor centrifuge is available at the clinical site, we suggest carrying out step 2.4 in a fixed-angle rotor at the same RCF as for a swingout head rotor but for only 10 minutes. This ensures that a PBMC layer is separated from the plasma, (see Figure 2B,C). While layering blood on a density gradient separation medium requires a brake-off centrifugation, blood in mononuclear cell preparation tubes can be centrifuged with brakes on due to the presence of a gel barrier in the tube that ensures the preservation of the PBMC layer separated from the denser blood components ${ }^{27,28}$. 
At least $4 \mathrm{~mL}$ of high quality, non-diluted plasma were obtained from the centrifugation of blood in the $8 \mathrm{~mL}$ tube format, which could be further clarified for its use in specialized analyses such as ctDNA sequencing or metabolomics studies ${ }^{29,30}$ (optional steps 4.1.1-4.1.4). The amount of isolated PBMCs, size of the pellets and hemolysis or red blood cell contamination have been other concerns coming from clinical sites and experience analyzing samples. The number of cells obtained from $8 \mathrm{~mL}$ blood was variable depending on the patient and disease setting, but in general the pellet obtained is small in size, and of a transparent/white coloration (Figure 3A,B). Due to these characteristics, it is important to visualize the pellet against a dark background to avoid its accidental aspiration during the wash steps (2.5 and 3.3). Sometimes pellets can have some red coloration due to red blood cell contamination (Figure 3C), and this has a negative effect on the quality of the preparation. To avoid losing small pellets such as those shown in Figure 3, transferring the PBMC pellet to a cryovial is facilitated by step 3.7 where the PBMC pellet is resuspended in $50 \mu \mathrm{L}$ of PBS.

The typical yield when using these tubes and blood from healthy individuals ranges between 7 to $21 \times 10^{6}$ cells for $8 \mathrm{~mL}$, and a cell recovery between 70 and $80 \%$ as it is our experience and as it has been previously shown ${ }^{27,28}$. This depends on both the individual cell counts and the operator and it is comparable to the cell numbers and cell recovery values obtained by other methods using density gradient (including systems utilizing tubes with a separation barrier $)^{15,27,28}$. An illustrative example of the variation on number of PBMCs isolated with this method depending on disease setting is the analysis of PBMC markers in chronic lymphocytic leukemia (CLL) patients. The number of cells recovered from an $8 \mathrm{~mL}$ mononuclear cell preparation tube when applying this protocol varied from $1.62 \times 10^{4}$ to $1.99 \times 10^{9}$ in 45 samples obtained from 7 patients in study NCT03328273 31 (Table 1). A related parameter is the protein concentration of the PBMC lysates obtained by this method, and this depends on the number of cells isolated and the efficiency of the protein extraction. The cell pellets lysed in section 6 were generated with RIPA buffer and sonication (step 6.6). The resuspension of the cell pellets in their same volume of lysis buffer usually results in a range of concentrations from 3 to $10 \mathrm{mg} / \mathrm{mL}$, and in this particular example the lysate concentrations were $6.8,8.3$ and $8.6 \mathrm{mg} / \mathrm{mL}$ for $0,0.2$ and $7 \mathrm{~Gy}$, respectively. However, this is subjected to a high variability when receiving samples from clinical sites that have not been optimally prepared, patient's disease, and the presence of hemoglobin from red blood cell contamination. For example, very small pellets need to be resuspended in a volume of lysis buffer larger than the cell pellet volume to allow for both protein concentration measurement and downstream biomarker analysis, resulting in more diluted samples. In such case, if a sample concentration is below $1 \mathrm{mg} / \mathrm{mL}$ this can pose a challenge to perform assays like western blot due to this high dilution factor. In contrast, in the CLL samples previously mentioned, the volume of lysis buffer added to resuspend the cell pellets varied from 50 to $500 \mu \mathrm{L}$, and protein concentration spanned from 1.62 to $19.77 \mathrm{mg} / \mathrm{mL}$ (Table 1).

When samples present red blood cell contamination or hemolysis (Figure $\mathbf{3 C}$ ), the protein concentration of the PBMC lysate becomes overestimated due to the inclusion of hemoglobin from the erythrocytes. This is the reason why one should do a visual inspection and annotation of such samples, as described in step 6.7 of the protocol. Loading sample in excess can compensate for the presence of hemoglobin when performing biomarker analysis as far as a loading 
control is included in the assay. Other more quantitative methods to measure hemolysis could be implemented, such as measuring absorbance at $414 \mathrm{~nm}^{32}$.

The DDR was analyzed in PBMCs obtained following this clinical protocol. To illustrate a situation that mimics DNA damaging clinical treatments such as radiotherapy or chemotherapy, whole blood from healthy volunteers was ex vivo subjected to $X$-ray radiation (Figure 4A). lonizing radiation (IR) such as X-rays induces different types of DNA damage, including DNA double-strand breaks (DSBs). DNA damage sensing of these lesions activates PIKKs such as ataxia-telangiectasia mutated (ATM), ATM and Rad3-related (ATR) and DNA-dependent protein kinase (DNA-PK), which engage DNA repair mechanisms like homologous recombination (HR) or non-homologous end joining (NHEJ). Activation of ATM by the presence of DSBs occurs by its recruitment to sites of damage by the MRN (MRE11-RAD50-NBS1) complex, causing ATM autophosphorylation at several residues including Ser1981. In turn, activated ATM phosphorylates the components of the MRN complex and other proteins such as histone variant $\mathrm{H} 2 \mathrm{AX}$ on Ser139 (where pSer139-H2AX is also known as $\mathrm{yH} 2 \mathrm{AX}$ ) to promote a structural change in the chromatin spanning from the DSB which facilitates the recruitment of other DDR factors ${ }^{33}$. PBMCs are responsive to ionizing radiation despite their low proliferation rate and mass spectrometry methods have allowed quantification of the upregulation of phosphorylated Ser635 on RAD50 by
ATM. This phosphorylation is reduced in the presence of ATM inhibitors and RAD50 pS635 has been further validated as a pharmacodynamic biomarker for clinical ATM inhibitor treatments in tumors by immunohistochemistry ${ }^{8}$. To evaluate the response of PBMCs to radiation, blood from healthy volunteers was subjected to different IR doses and samples were collected after a $1 \mathrm{~h}$ incubation at $37^{\circ} \mathrm{C}$ (Figure 4A). For this purpose, bloods were transferred to plastic tubes to avoid reducing the yield of the PBMC isolation due to high temperature (step 6.2). We analyzed how ATM was activated not only by looking at the previously reported RAD50 pS635 but also ATM pS1981 and yH2AX. In the three cases examined an increase in these post-translational modifications was observed at higher IR doses (Figure 4B). Interestingly, the phosphorylation of ATM and RAD50 was substantial at the low dose of $0.2 \mathrm{~Gy}$, which suggests these post-translational modifications may be feasibly interrogated as PD biomarkers for treatments involving the generation of DNA DSBs with a good dynamic range, not only in tumor samples but in peripheral blood. This allows the monitoring of the PD response to the treatment by acquiring longitudinal samples through the course of treatment. The timing from the blood draw to the processing of the samples is critical to ensure these signaling cascades are still active as delays in processing will impact on the kinetics of such cascades and one could miss the phosphorylation events used as phosphomarkers. 
A)

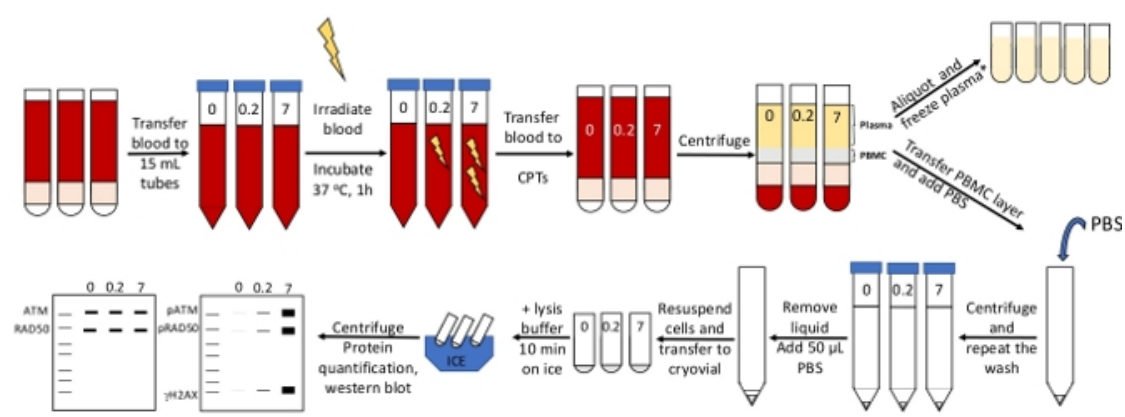

B)

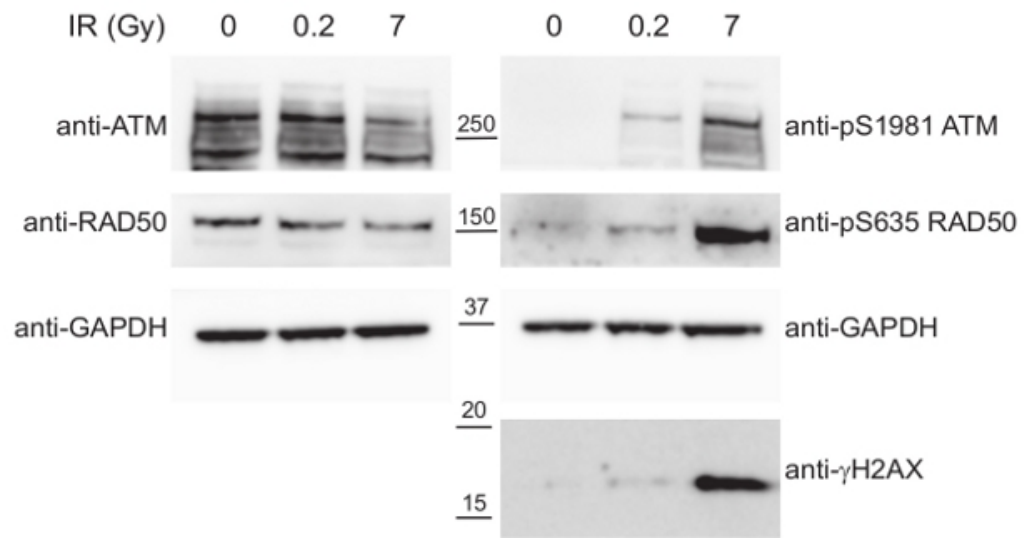

Figure 4: PBMCs isolated from ex vivo irradiated blood following the present clinical protocol display biomarkers to inform on the extent of the DNA damage caused by the treatment. (A) Schematic overview of the protocol for the preparation of PBMCs and analysis of the DDR upon whole-blood irradiation. *2 extra centrifugation steps required for ctDNA analysis/metabolomics. (B) Western blot showing dose-dependent upregulation of DDR phospho-biomarkers in PBMCs. Please click here to view a larger version of this figure. 


\begin{tabular}{|c|c|c|c|}
\hline Sample & $\begin{array}{l}\text { Number of PBMCs/ } \\
\qquad 8 \mathrm{~mL} \text { blood }\end{array}$ & $\begin{array}{l}\text { Supplemented RIPA } \\
\text { buffer volume }(\mu L)\end{array}$ & $\begin{array}{l}\text { Final concentration } \\
\qquad(\mathrm{mg} / \mathrm{mL})\end{array}$ \\
\hline A. 1 & 39100000 & 70 & 12.16 \\
\hline A. 2 & 97400000 & 100 & 4.54 \\
\hline A. 3 & 233000000 & 150 & 7.63 \\
\hline A. 4 & 316000000 & 150 & 16.87 \\
\hline A. 5 & 387000000 & 150 & 12.60 \\
\hline A. 6 & 459000000 & 150 & 12.71 \\
\hline A. 7 & 414000000 & 200 & 15.67 \\
\hline A. 8 & 253000000 & 150 & 14.56 \\
\hline A. 9 & 509000000 & 300 & 10.67 \\
\hline B. 1 & 15200000 & 70 & 2.96 \\
\hline B. 2 & 10500000 & 50 & 4.59 \\
\hline B. 3 & 13200000 & 50 & 3.99 \\
\hline B. 4 & 34800000 & 100 & 10.41 \\
\hline B. 5 & 1620000 & 70 & 7.11 \\
\hline B. 6 & 70200000 & 100 & 9.26 \\
\hline B.7 & 65400000 & 100 & 12.10 \\
\hline B. 8 & 91100000 & 150 & 11.82 \\
\hline C.1 & 6330000 & 70 & 4.04 \\
\hline C.2 & 4400000 & 150 & 19.77 \\
\hline C. 3 & 68000000 & 100 & 8.96 \\
\hline C. 4 & 35100000 & 50 & 9.30 \\
\hline C. 5 & 35400000 & 70 & 10.55 \\
\hline C.6 & 99200000 & 100 & 16.19 \\
\hline D.1 & 402000000 & 70 & 7.23 \\
\hline D.2 & 826000000 & 300 & 16.95 \\
\hline D.3 & 1990000000 & 300 & 14.87 \\
\hline
\end{tabular}




\begin{tabular}{|c|c|c|c|}
\hline D.4 & 1000000000 & 300 & 18.34 \\
\hline D.5 & 1160000000 & 400 & 16.13 \\
\hline D.6 & 806000000 & 400 & 19.40 \\
\hline E.1 & 302000000 & 300 & 13.86 \\
\hline E.2 & 990000000 & 500 & 19.04 \\
\hline E.3 & 1200000000 & 400 & 17.13 \\
\hline F.1 & 4010000 & 50 & 1.62 \\
\hline F.2 & 5170000 & 50 & 2.84 \\
\hline F.3 & 2810000 & 50 & 3.69 \\
\hline F.4 & 3700000 & 75 & 3.62 \\
\hline F.5 & 3460000 & 70 & 4.03 \\
\hline F.6 & 7060000 & 50 & 3.32 \\
\hline G.1 & 60700000 & 70 & 6.57 \\
\hline G.2 & 82100000 & 150 & 7.78 \\
\hline G.3 & 30500000 & 70 & 8.28 \\
\hline G.4 & 134000000 & 100 & 15.14 \\
\hline G.5 & 91900000 & 100 & 8.61 \\
\hline G.6 & 372000000 & 150 & 15.88 \\
\hline G.7 & 574000000 & 200 & 15.01 \\
\hline \multicolumn{4}{|c|}{ Longitudinal PBMC samples corresponding to 7 patients } \\
\hline
\end{tabular}

Table 1: Cell number and protein concentration of PBMC pellets from chronic lymphocytic leukemia patients (CLL).

The data presented in this table correspond to 45 PBMC samples from 7 CLL patients participating in study NCT03328273 collected in mononuclear cell preparation tubes. These are original data generated using samples from the cited study ${ }^{31}$.

\section{Supplementary File: Alternative protocol options. Please} click here to download this file.

\section{Discussion}

High-quality preparations of PBMCs and plasma that can be robustly and reproducibly prepared at clinical trial sites are invaluable to inform clinical trial peripheral predictive and pharmacodynamic translational biomarker endpoints. Here 
we have provided a short, clear protocol that addresses the typically problematic steps that have been heretofore vulnerable to execution errors in a clinical trial setting. However, the protocol can be further optimized to meet specific requirements, such as time constraints at the clinical site or type of downstream analyses (see Supplementary File).

To this aim, we have shown how to isolate both PBMCs and plasma from whole blood using mononuclear cell preparation tubes to produce frozen PBMC pellets and frozen plasma suitable for a variety of downstream analyses. We have called attention to particularly critical protocol steps involving centrifugation and identification of the PBMC layer in step 2.5, and PBMC pellets in steps 3.3 and 3.6. Historically, where clinical sites have often gone wrong is in setting the centrifuge to the correct units (confusing an RCF or $x g$ value with an RPM value), delaying the processing of blood samples, temperature and the presence of large volumes of PBS above the frozen cell pellet. In most centrifuge rotors erroneously entering a $x g$ value as an RPM setting will result in significant under-centrifugation with a resulting poorly defined or absent PBMC layer, and potential inadvertent PBMC discarding during wash steps due to inefficient cell pelleting. However, there is a possibility that a PBMC layer is not visible despite using the right centrifugation settings and rotor adaptor if the patient has developed leukopenia. This condition can affect patients enrolled in oncology trials because of chemotherapy or radiation therapy and should be considered. Another critical point that has been made clear in the protocol is that samples must be processed within 1-2 $\mathrm{h}$ from the blood draw to decrease the possibility of hemolysis negatively impacting the protocol. Furthermore, aiming to process the samples during the first hour of the blood draw reduces ex vivo variability, which can have a great impact in pharmacokinetics readouts and on biomarkers affected by blood preservation or active signaling pathways, such as the case shown in Figure 4. Delays in sample processing can also have a detrimental effect in cell viability if cells are going to be cryopreserved ${ }^{34}$. Another factor that can affect both the yield and red blood cell contamination is the storage and centrifugation temperature, which should be kept at room temperature $\left(18-25^{\circ} \mathrm{C}\right)$. Lower temperatures increase the density of the density gradient medium, which results in a higher degree of red blood cell and granulocyte contamination as these cells do not aggregate as well. On the other hand, higher temperatures lead to PBMCs trapped between aggregated erythrocytes, hence reducing the yield of the preparation ${ }^{15,27,28}$. And finally, it is crucial that no more than $50-100 \mu \mathrm{L}$ of liquid are present with the cell pellet in the cryovial, as this negatively impacts the concentration of any protein lysates obtained in downstream processing of these PBMC preparations. An excess of liquid will overdilute samples, leading to lysates with very low protein concentration not suitable for biomarker analysis. In addition, preservation of any post-translational modifications will be impaired, and the efficiency of the lysis will be also greatly reduced.

Mononuclear cell preparation tubes were chosen as they offer the most straightforward way to isolate both PBMCs and plasma in a single blood draw for clinical trials with, in our experience, excellent reproducibility. The blood processing does not require highly trained operators, and the use of a single tube removes the need of diluting the blood and its transfer to a different tube, lowering the hazard risk; shortens the protocol due to performing the centrifugation steps with brakes on; and all reagents are in the tube, which reduces variability. In our experience, these benefits outweigh the higher cost of these tubes when compared to other classical methods comprising only the use of a density 
gradient separation medium ${ }^{27,28}$ (£ 410 per 60 units while lymphoprep medium for $6650-\mathrm{mL}$ preparations is $£ 215$ ). They are available in two types of anticoagulants, heparin and citrate, both of which are comparable at maintaining functionality of the isolated PBMCs ${ }^{35}$, therefore, the choice of one anticoagulant over the other will be based on possible influence of heparin or citrate in the downstream biomarker studies. While it has been shown that EDTA tubes provide the highest PBMC isolation yield compared to heparin or citrate ${ }^{13}$, the benefit of ease of use of the onetube-only manipulation counterbalances this consideration. If cytokines are going to be analyzed anti-coagulants can have an effect of the levels detected in plasma, hence both anticoagulants should be tested before selecting one for the clinical trial ${ }^{36}$. If the plasma is going to be used for metabolomics studies, using heparin as anticoagulant would be preferred ${ }^{37}$. Therefore, the only point left to the end user or clinical trial translational scientist is whether citrate or heparin will be more appropriate for their purposes once costs have been assessed.

While the benefits of using cell preparation tubes are numerous compared to the limitations they pose (higher cost and availability of a restricted range of anticoagulants), the main limitation of the use of PBMCs or plasma to obtain PD biomarkers in clinical trials, especially in oncology, can be unrelated to the isolation method. Except for hematological cancers, where tumor is directly sampled from peripheral blood, for other cancer indications plasma and PBMCs are surrogate tissues which do not necessarily mimic the primary tumor. Peripheral tissue may not share the genome and epigenome with the primary tumor, therefore, the peripheral analysis of biomarkers dependent on a specific tumor mutation is mainly limited to ctDNA analysis (from plasma) or CTCs (by subsequent sorting of the PBMC layer). In addition, signaling cascades driving or contributing to the tumor proliferation may not be as active in peripheral blood. This challenge can be overcome by applying biomarker discovery approaches targeting blood ${ }^{8}$ to identify alternative biomarkers or coupling ex vivo treatments to the isolation of plasma $^{38}$ and PBMC preparations ${ }^{26}$.

In the current protocol frozen PBMC pellets can be easily processed off the clinical site to give protein lysates which can be evaluated by western blotting or ELISA techniques. Alternative methods to use PBMCs to enable IHC methods have also been presented (Supplementary File). In addition, we have also detailed the possibility of cryopreserving PBMCs (see Supplementary File) for immune cell monitoring, a relevant application in oncology, with immune checkpoint inhibitors and ADCs increasingly tested in clinical trials. The assessment of immune functions such as $A D C C^{16}$ and immunophenotyping are applications compatible with cryopreserved PBMCs isolated from mononuclear cell preparation tubes ${ }^{15}$. There is a caveat on cryopreservation, as it can promote down-regulation of certain surface and internal markers and might impair certain cell functions, however PBMC cryopreservation is the only feasible way to perform these assays due to time constraints when handling samples from multiple clinical sites to the processing in external labs ${ }^{14,15}$, and these detrimental effects can be greatly overcome by good thawing methods and resting periods ${ }^{39}$

In conclusion, the protocol provided here will allow the dependable preparation of PBMCs and plasma samples in any clinical institution with common equipment and materials so that translational endpoints from peripheral blood can be robustly enabled in global clinical trials. 
Finally, we demonstrate how the analysis of PBMC lysates can mechanistically inform the response to DNA-damaging agents by showing a dose-dependent post-translational modification of key DDR factors, which can be used to help shape clinical development. Forward-looking, implementation of methods that are more quantitative than western blotting (e.g., mass spectrometry ${ }^{40}$ ) and require less input material (such as capillary western blotting and ELISA) would help to move these preclinical results towards a more robust, systematic evaluation of PBMC patient samples.

\section{Disclosures}

All the authors are or were employees and stake holders of AstraZeneca. VM is an employee of Acerta Pharma, owns stock in AstraZeneca and Gilead Sciences.

\section{Acknowledgments}

We would like to thank all the members of Translational Medicine at AstraZeneca Oncology Research and Early Development for their feedback on the protocol, especially Hedley Carr, Tammie Yeh and Nathan Standifer for advice on plasma preparation for ctDNA analysis, on PBMC isolation, and PBMC cryopreservation and immunophenotyping, respectively.

\section{References}

1. Hoelder, S., Clarke, P. A., Workman, P. Discovery of small molecule cancer drugs: successes, challenges. and opportunities. Molecular Oncology. 6, 155-176 (2012).

2. Harrigan J. A., Jacq, X., Martin, N. M., Jackson, S. P. Deubiquitylating enzymes and drug discovery: emerging opportunities. Nature Reviews Drug Discovery. 17, 57-77 (2018).
3. Brown, J. S., O'Carrigan, B., Jackson, S. P., Yap, T. A. Targeting DNA repair in cancer: beyond PARP inhibitors. Cancer Discovery. 1, 20-37 (2017).

4. Pettersson, M., Crews C. M. PROteolysis TArgeting Chimeras (PROTACs)-Past, present and future. Drug Discovery Today: Technologies. 31, 15-27 (2019).

5. Scott, A. M., Wolchok, J. D., Old, L. J. Antibody therapy of cancer. Nature Reviews Cancer. 12, 278-287 (2012).

6. Thomas, A., Teicher B. A., Hassan, R. Antibody-drug conjugates for cancer therapy. The Lancet Oncology. 17 (6), e254-e252 (2016).

7. Ferguson, F. M., Gray, N. S. Kinase inhibitors: the road ahead. Nature Reviews Drug Discovery. 17 (5), 353-377 (2018).

8. Jones, G. N. et al. pRAD50: a novel and clinically applicable pharmacodynamic biomarker of both ATM and ATR inhibition identified using mass spectrometry and immunohistochemistry. British Journal of Cancer. 119 (10), 1233-1243 (2018).

9. Cook, D. et al. Lessons learned from the fate of AstraZeneca's drug pipeline: a five-dimensional framework. Nature Reviews Drug Discovery. 13, 419-431 (2014).

10. Overman, M. J. et al. Use of research biopsies in clinical trials: are risks and benefits adequately discussed? Journal of Clinical Oncology. 31 (1), 17-22 (2012).

11. Olson, E. M., Lin, N. U., Krop, I. E., Winer, E. P. The ethical use of mandatory research biopsies. Nature reviews Clinical Oncology. 8, 620-625 (2011).

12. O'Donnell, A. et al. Phase I pharmacokinetic and pharmacodynamic study of the oral mammalian target of rapamycin inhibitor Everolimus in patients with advanced 
solid tumors. Journal of Clinical Oncology. 26 (10), 1588-1595 (2008).

13. Fong, P. C. et al. Inhibition of Poly(ADP-Ribose) polymerase in tumors from $B R C A$ mutation carriers. The New England Journal of Medicine. 361 (2), 123-134 (2009).

14. Verschoor, C. P., Kohli, V., Balion, C. A comprehensive assessment of immunophenotyping performed in cryopreserved peripheral whole blood. Cytometry $B$ Clinical Cytometry. 94 (5), 662-670 (2018).

15. Ruitenberg, J. J. et al. VACUTAINER $® C P T^{\mathrm{TM}}$ and Ficoll density gradient separation perform equivalently in maintaining the quality and function of PBMC from HIV seropositive blood samples. BMC Immunology. 7 (11), (2006).

16. Yamashita, M. et al. A novel method for evaluating antibody-dependent cell-mediated cytotoxicity by flowcytometry using cryopreserved human peripheral blood mononuclear cells. Scientific Reports. 6 (19772), 1-10 (2016).

17. Schiavon, G. et al. Analysis of ESR1 mutation in circulating tumor DNA demonstrates evolution during therapy for metastatic breast cancer. Science Translational Medicine. 7 (313), 313ra182 (2015).

18. Lee, J. et al. Tumor genomic profiling guides metastatic gastric cancer patients to targeted treatment: the VIKTORY umbrella trial. Cancer Discovery. CD-19-0442 (2019).

19. Abbosh, C. et al. Phylogenetic ctDNA analysis depicts early-stage lung cancer evolution. Nature. 545, 545-451 (2017).
20. Thress, K. S. et al. Acquired EGFR C797S mutation mediates resistance to AZD9291 in non-small cell lung cancer harboring EGFR T790M. Nature Medicine. 21 (6), 560-562 (2015).

21. Rossi, G., Ignatiadis, M. Promises and pitfalls of using liquid biopsy for precision medicine. Cancer Research. 79 (11), 2798-2804 (2019).

22. Balasubramanian, P. et al. Isolation and characterisation of circulating tumor cells (CTCs) from peripheral blood specimens of patients with advanced solid tumor malignancies (using ApoStream $®$ instrumentation) [abstract 3062]. Proceedings of the Annual meeting of the American Association for Cancer Research. San Diego, CA (2014).

23. Qin, J., Alt, J. R., Hunsley, B. A., Williams, T. L., Fernando, M. R. Stabilization of circulating tumor cells in blood using a collection device with a preservative reagent. Cancer Cell International. 14 (13), 1-6 (2014).

24. Biomarkers definitions working group. Biomarkers and surrogate endpoints: preferred definitions and conceptual framework. Clinical Pharmacology \& Therapeutics. 69 (3), 89- 95 (2001).

25. Crowley, E., Di Nicolantonio, F., Loupakis, F., Bardelli, A. Liquid biopsy: monitoring cancer-genetics in the blood. Nature Reviews Clinical Oncology. 10, 472-484 (2013).

26. Bundred, N. et al. Evaluation of the pharmacodynamics of the PARP inhibitor olaparib: a phase I multicentre trial in patients scheduled for elective breast cancer surgery. Investigational New Drugs. 31, 949- 958 (2013).

27. Rosado, M. et al. Advances in biomarker detection: Alternative approaches for blood-based biomarker 
detection. Advances in Clinical Chemistry. 92, 141-199 (2019).

28. Grievink, H. W. et al. Comparison of three isolation techniques for human peripheral blood mononuclear cells: cell recovery and viability, population composition and cell functionality. Biopreservation and Biobanking. 14 (5), 410- 415 (2016).

29. Khadka, M. et al. The effect of anticoagulants, temperature, and time on the human plasma metabolome and lipidome from healthy donors as determined by liquid chromatography-mass spectrometry. Biomolecules. 9 (5), 200: 1-15 (2019).

30. Hellmann, M. D., et al. Circulating tumor DNA analysis to assess risk of progression after long-term response to PD-(L)1 blockade in NSCLC. Clinical Cancer Research. 26 (12), 2849-2858 (2020).

31. ClinicalTrials.gov [Internet]. Identifier NCT03328273, A study of AZD6738 and Acalabrutinib in subjects with relapsed or refractory chronic lymphocytic leukemia (CLL); National Library of Medicine (US). Bethesda (MD). Available from https://clinicaltrials.gov/ct2/show/ NCT03328273 (2017).

32. Kirschner, M. B. et al. The impact of hemolysis on cellfree microRNA biomarkers. Frontiers in Genetics. 4 (94), $1-13(2013)$.

33. Blackford, A. N., Jackson, S. P. ATM, ATR, and DNA-PK: The Trinity at the Heart of the DNA Damage Response. Molecular Cell. 66, 801-817 (2017).

34. Riedhammer, C., Halbritter, D., Weissert, R. Peripheral Blood Mononuclear Cells: Isolation, Freezing, Thawing, and Culture. Methods in Molecular Biology. 1304, 53-61 (2016).
35. Basavaraj, M. G., Østerud, B., Hansen, J. B. Influence of different anticoagulants on monocyte procoagulant functions and monocyte-platelet aggregates formation. Journal of Thrombosis and Haemostasis. 9 (8), 16731676 (2011).

36. Biancotto, A., Feng, X., Langweiler, M., Young, N. S., McCoy, J. P. Effect of anticoagulants on multiplexed measurements of cytokine/chemokines in healthy subjects. Cytokine. 60, 438-446 (2012).

37. Wawrzyniak, R. et al. New plasma preparation approach to enrich metabolome coverage in untargeted metabolomics: plasma protein bound hydrophobic metabolite release with proteinase K. Scientific Reports. 8, 1-10 (2018).

38. Duffy, D. et al. Standardized whole blood stimulation improves immunomonitoring of induced immune responses in multi-center study. Clinical Immunology. 183, 325-335 (2017).

39. Wang, L. et al. Standardization of cryopreserved peripheral blood mononuclear cells through a resting process for clinical immunomonitoring- development of an algorithm. Cytometry A Clinical Cytometry. 89, 246-258 (2016).

40. Whiteaker, J. R. et al. Targeted mass spectrometry enables robust quantification of FANCD2 monoubiquitination in response to DNA damage. DNA Repair. 65, 47-53 (2018).

41. Lam, N. Y., Rainer, T. H., Chiu, R. W., Lo, Y. M. EDTA is a better anticoagulant than heparin or citrate for delayed blood processing for plasma DNA analysis. Clinical Chemistry. 50, 256-257 (2004). 
42. Parpart-Li, S. et al. The effect of preservative and temperature on the analysis of circulating tumor DNA.

Clinical Cancer Research. 23 (10), 2471-2477 (2017). 